\title{
Environmental Stability of Intercalated Graphite Fibers
}

James R. Gaier and Donald A. Jaworske

Lewis Research Center

Cleveland, Ohio

\section{LBRARY GOPY}

May 1985
$.1 . .21885$

DANGLY RESEARCH CENTER

MRRARY NASA

MNMTOM MRCINIA 


\title{
ENVIRONMENTAL STABILITY OF INTERCALATED GRAPHITE FIBERS
}

\author{
J.R. Gajer and D.A. Jaworske \\ National Aeronautics and Space Administration \\ Lewis Research Center \\ Cleveland, Ohio 44735
}

\begin{abstract}
SUMMARY
Graphite fibers intercalated with bromine, fodine monochloride, ferric chloride, and cupric chloride were subjected to stability tests under four environments which are encountered by engineering materials in the aerospace industry: ambient laboratory conditions, as would be experienced during handling operations and terrestrial applications; high vacuum, as would be experienced in space applications; high humidity, as would be experienced in marine applications; and high temperature, as would be experienced in some processing steps and applications. Monitoring the resistance of the fibers at ambient laboratory conditions revealed that only the ferric chloride intercalated fibers were unstable, due to the absorption of water from the air. All four types of intercalated fibers were stable for long periods under high vacuum. Ferric chloride, cupric chloride, and lodine monochloride intercalated fibers were sensitive to high humidity conditions. All intercalated fibers began to degrade above $250{ }^{\circ} \mathrm{C}$. The order of their thermal stability, from lowest to highest. was cupric chloride, iodine monochloride, bromine, and ferric chloride. Of the four types of intercalated fibers tested, the bromine intercalated fibers appear to have the most potential for application, based on environmental stability.
\end{abstract}

\section{INTRODUCTION}

In many aerospace applications the low mass density and high strength of graphite fibers make their composites an attractive alternative to other materials. Problems in some applications, however, have resulted because these composites are relatively poor electrical conductors. For example their use in eletromagnetic interference shielding for electronic equipment now requires additional metallic cabinets or coatings. Composite spacecraft and aircraft structures cannot be used as electrical grounding buses, so metallic structures must be added. A final example is aircraft skin, which requires a thin layer of metal to be placed over the composite structure to provide sufficient lightning strike survivability. One way to improve the conductivity of the composites is to improve the conductivity of the embedded graphite fibers by intercalation. A variety of chemical reagents are suitable for graphite intercalation, but the vast majority of those which improve the electrical conductivity of graphite are unstable under ambient conditions. The purpose of this study was to compare the environmental stability of four reportedly air stable (refs. 1 to 4) graphite intercalants: bromine, iodine monochloride, ferric chloride, and cupric chloride.

The electrical resistances of the four types of intercalated graphite fibers were continually monitored while the fibers were subjected to four environments which would be encountered by engineering materials in the aerospace industry. 
The first test was at ambient laboratory conditions. Industrial acceptance of a new material is much more likely if it does not require special handling. Also, materials that outgas noxious substances (such as chloride or bromine) are not likely to be accepted. Regardless of the final application, intercalated fibers will undoubtedly be exposed to ambient conditions for indefinite lengths of time, until needed in a particular processing scheme.

Any space bound material must be stable in high vacuum. Not only is this important to maintain the structural properties of the composite, but also to avoid the use of materials that outgas corrosive substances which could damage other spacecraft components. A recent report that nitric acid intercalated graphite is unstable under vacuum (ref. 5) emphasizes the importance of this investigation.

High humidity tests were included because in some applications spacecraft and aircraft may be exposed to high humidity or moisture. Both ferric chloride and cupric chloride intercalated graphite, long thought of as stable graphite intercalation compounds, have recentiy been found susceptible to water vapor (refs. 6 and 7 ). The humidity tests were conducted in this study to determine the extent of this susceptibility.

The last test, high temperature, is important because intercalated graphite fibers must survive the many processes that require high temperatures (molding operations, curing, etc.). Also, many applications require long-term high-temperature stability. In addition to being an important environmental test, high-temperature studies can provide data which shed light on the mechanisms of deintercalation.

\section{METHODS AND MATERIALS}

Pitch-based Union Carbide P-100 fibers were chosen for this study because of their good mechanical and electrical properties (ref. 8 ) and because they were commercially avallable in spools. Before the fibers were intercalated they were heated to $350{ }^{\circ} \mathrm{C}$ in air for $24 \mathrm{hr}$ to remove a polyvinyl alcohol sizing that had been added by the manufacturer to improve handing.

The bromine intercalation was carried out at room temperature. The fibers were sealed in a small glass bell jar with a bromine (Fisher Scientific) reservoir and allowed to react for about $24 \mathrm{hr}$. The vapor pressure of the bromine at room temperature was estimated to be $22 \mathrm{kPa}$ (165 torr) (ref. 9). Studies on single fibers showed that the reaction was complete within about $4 \mathrm{hr}$. The average resistivity for the bromine intercalated fibers was $52 \mu \Omega-\mathrm{cm}$.

The iodine monochloride intercalation was carried out at $58{ }^{\circ} \mathrm{C}$. The fibers and an iodine monochloride (Eastman Kodak) reservoir were sealed in a quartz tube and placed in tube furnace for $18 \mathrm{hr}$. The vapor pressure of iodine monochloride was estimated to be about $22 \mathrm{kPa}$ (165 torr) (ref. 9). Intercalation was judged complete by analogy with the bromine reaction, because the final resistivity values of the lodine monochloride intercalated fibers $(46 \mu \Omega-\mathrm{cm})$ were similar to those of the bromine intercalated fibers. 
The ferric chloride intercalation was performed in an all-glass reaction vessel placed in a tube furnace. The ferric chloride (AESAR, anhydrous, 98 percent) was dried at $100{ }^{\circ} \mathrm{C}$ overnight under vacuum. To initiate the reaction, the furnace was heated to $260{ }^{\circ} \mathrm{C}$, and chlorine gas was added. After $1 \mathrm{hr}$ the furnace was turned off and allowed to cool. The reaction vessel was purged with nitrogen before the fibers were removed. Then the fibers were placed in air tight dishes and placed in a dessicator filled with calcium sulfate. To separate the fibers so that they could be mounted on the sample holders, a small group of fibers was quickly washed with water and air dried. The resistivity of the ferric chloride intercalated fibers in this state varied widely but averaged about $130 \mu \Omega-\mathrm{cm}$.

The cupric chloride intercalated fibers were prepared in all-glass ampoule. The fibers were placed in the ampoule with anhydrous cupric chloride (NOAH Chemica1). One fiber was mounted on an embedded four-point probe to monitor the progress of the reaction. After heating under vacuum for several hours at $100{ }^{\circ} \mathrm{C}$ to remove any residual water, the glass ampoule was sealed and heated incrementally to $480^{\circ} \mathrm{C}$ to initiate sublimation of the cupric chloride. The ampoule was kept at $480^{\circ} \mathrm{C}$ for $\sim 16 \mathrm{hr}$. Before cooling, the temperature was raised an additional $60^{\circ} \mathrm{C}$ over a period of $2 \mathrm{hr}$, but no significant increase in conductivity was observed in the fiber mounted on the embedded four-point probe. After cooling to room temperature, the ampoule was opened, and the intercalated fibers were removed. While there was a wide variation in the fiber resistivity values, the average value was about $20 \mu \Omega-\mathrm{cm}$.

Individual fibers were mounted with carbon paint on sample holders which contained four-point probe contacts. Two types of sample holders were used: one with copper contacts on an integrated circuit board (fig. 1) and a second with the same geometry, but made from platinum sputtered on an aluminum oxide substrate. The latter had the oxidation resistance necessary for the hightemperature tests.

Resistance measurements were made using a constant current source (Keithiy model 225) to supply a constant $d c$ current to the fiber through the outer contacts, while measuring the voltage drop between the inner contacts (Keithiy model $181 \mathrm{nVm})$. Voltage measurements were obtained for both polarities, and the absolute value of the $2 \mathrm{~V}$ was then averaged to cancel out electrochemical and electrothermal effects. All measurements were made with a current of $100 \mu \mathrm{A}$. Voltages were measured to the nearest $0.01 \mathrm{mV}$, giving a relative uncertainty of about 0.2 percent.

For the ambient conditions tests, fibers were mounted on the copper sample holders and stored in microscope slide racks in a laboratory drawer. No attempt was made to control either the temperature or the humidity except for the building air conditioning. Temperatures ranged from about 16 to $26{ }^{\circ} \mathrm{C}$, and the relative humidity ranged widely. The resistance of the fibers was measured at first hourly and then at increasing time intervals until, after several weeks, the measurements occurred monthiy.

The vacuum tests were carried out by placing fibers mounted on the copper sample holders in a bell jar which was sustained at a vacuum of at least $1.33 \times 10^{-7} \mathrm{~Pa}\left(10^{-5}\right.$ tor $)$. The fibers were removed from the bel1 jar at regular intervals, and their resistance measured. Since the ferric chloride samples proved to be the most sensitive to water vapor, they were always 
measured first to minimize the amount of water picked up from the air. Effort was made to minimize the exposure of the fibers to ambient conditions during the measurements.

The high humidity environment was created by setting a small beaker of water and a copper sample holder within a larger beaker. It was then sealed with Parafilm and placed in an incubator maintained at $60^{\circ} \mathrm{C}$. Each sample thus had its own individual chamber, preventing cross-contamination, but all were stored in the same incubator, ensuring uniform temperature. At regular intervals the sample holders were removed from the sealed beakers and allowed to cool to room temperature. After the resistances were measured, the sample holders were promptly returned to the humidity chambers before a full equilibrium with the room humidity could be established.

Experiments in the temperature regime up to $150{ }^{\circ} \mathrm{C}$ were carried out in an oven (in air at $1 \mathrm{~atm}$ pressure), which could maintain a temperature from 60 to $150^{\circ} \mathrm{C}$ to within $1^{\circ}$. Copper sample holders were used for these tests (though they discolor within a few days at $\left.150^{\circ} \mathrm{C}\right)$. Higher temperature tests were carried out in large-thermal-mass muffle furnaces which could maintain temperatures above $200{ }^{\circ} \mathrm{C}$ to within $1^{\circ}$. The platinum-aluminum oxide sample holders were used for these tests to avoid oxidation of the sample holder and to maintain good electrical contact. In all cases the sample holders were removed from the ovens and brought to room temperature before fiber resistance measurements were made.

Rather than using the freshly intercalated fibers, several days were allowed to elapse before the tests were initiated. Thus, the initial state of the fibers for this study was the room-temperature intercalated fiber after it had come to equilibrium with the atmosphere. Ferric chloride intercalated fibers, however, gradually absorbed water from the air over a period of months. Therefore, water was removed from these fibers by placing them in a $200{ }^{\circ} \mathrm{C}$ oven overnight before conducting either the humidity or high temperature tests. The average resistivity of the ferric chloride intercalated fibers when dried using this procedure was about $75 \mu \Omega-\mathrm{cm}$.

\section{RESULTS AND DISCUSSION}

\section{Ambient Conditions}

To separate the long-term changes in resistivity at ambient conditions from those associated with the initial establishment of equilibrium after intercalation, several days were allowed to elapse before data were taken. For bromine this interval was 12 days, for lodine monochloride 13 days, for ferric chloride 16 days, and for cupric chloride 17 days.

Figure 2 shows the time dependence of the resistance ratio (final intercalated fiber resistance to initial intercalated fiber resistance) for the four types of intercalated fibers. It reveals that the resistivity of the bromine intercalated fibers did not change for the duration of the tests, which is well over a year at the time of this writing.

Iodine monochloride intercalated fiber resistivity rose by about 1 percent over its initial value, which was probably within the uncertainty of the measurement. 
While the ambient conditions tests of cupric chloride intercalated fibers were only in their early stages, there appeared to be a slow degradation with time (about 2 percent over a month). This was probably associated with the uptake of water from the atmosphere (see fig. 4).

The effect of atmospheric water was seen more dramatically in the ferric chloride intercalated fibers (fig. 2). They were very sensitive to moisture, as will be established later, and that sensitivity was the cause of the longterm instability in the resistance of these fibers. It is worth noting that after a long period of time (about 200 days), the resistance ratio of the ferric chloride intercalated fibers leveled off at a value of about 1.45 . Although this resistivity was comparable with that of the pristine fibers, it was reversible and so not a consequence of deintercalation. At that point the fibers were probably in a stable equilibrium with atmospheric moisture. Also note the large fiber to fiber variation, represented by the vertical lines at each data point. Auger depth profliing indicated that these fibers were not intercalated completely through, but only in a thin anular ring (ref. 10). Variations in fiber resistivity and resistance ratio changes were probably associated with variations in the amount of ferric chloride present in each fiber.

\section{Vacuum Effects}

Dziemianowicz and Forsman reported that, on exposure to vacuum, neutral nitric acid molecules were removed from nitric acid intercalated, highly oriented pyrolytic graphite (HOPG) and that the carbon lattice was partially oxidized to carbon monoxide and carbon dioxide (ref. 5). The result was a degradation of both the carbon lattice and the intercalation compound. All four of the intercalants tested in this study were found to be stable under vacuum at room temperature (fig. 3). These results are in sharp contrast to the nitric acid results of Dziemianowicz and Forsman.

\section{Humidity Effects}

The effects of humidity on the resistance of the intercalation compounds are shown in figure 4 . The bromine intercalated fibers were totally unaffected for at least 10 months in 100 percent humidity at $60^{\circ} \mathrm{C}$. This was tested in the extreme by submerging bromine intercalated fibers in tap water. There were no changes in the resistance of the submerged fibers after 8 months.

Figure 5 shows the effect of water vapor at $60^{\circ} \mathrm{C}$ on the resistance ratio of lodine monochloride intercalated fibers. Also shown in that figure is the effect of $60{ }^{\circ} \mathrm{C}$ temperature on the resistance ratfo of the same fibers in the absence of water vapor. While some increase in resistance appears to be from the higher temperature, most of the increase must be attributed to the water vapor.

The effects of the $60^{\circ} \mathrm{C}, 100$ percent humidity environment were most dramatic with the cupric chloride and ferric chloride intercalated fibers. This is illustrated by the dragration of the resistance ratio on exposure to a high humity environment for both cupric chloride and ferric chloride intercalated fibers (fig. 4) and, converes ly, by the improvement in the resistance ratio of the ferric chloride fibers when they were moved from 
ambient conditions and placed in various drying environments (fig. 6). The results from these experiments provide compeling evidence suggesting that the uptake and loss of water is a reversible process and that the steadily increasing resistance ratio of ferric chloride intercalated fibers was not due to deintercalation. This sensitivity to humidity presents a severe limitation for the use of these fibers as engineering materials.

Several techniques for drying the ferric chloride intercalated fibers were tested for speed and effectiveness. The results shown in figure 6 suggest that water is not totally removed by a vacuum, nor with a calcium sulfate dessicant, but that heat must be applied. Even when the fibers were heated to $200{ }^{\circ} \mathrm{C}$ for $15 \mathrm{hr}$, some evidence of water remained, as indicated by a slight dip in the resistance when the fibers were further heated to 250 or $300{ }^{\circ} \mathrm{C}$ (see fig. 10). Since both ferric chloride and cupric chloride crystals are highly hygroscopic, it is tempting to suggest that intercalants which are themselves hygroscopic form hygroscopic intercalation compounds.

\section{Temperature Effects}

The thermal stability of these materials was evaluated for long periods (hours to weeks). While the thermal stability for some intercalants has been investigated before (ref. 4), the samples were heated to high temperatures for only a few minutes. Curing times for graphite fiber/resin composites are typically on the time scale of hours.

Figures 7 to 11 show the resistance ratio as a function of time for pristine fibers and for each type of intercalated fiber at several different temperatures. The highest temperature at which a compound exhibits long-term stability (i.e., $24 \mathrm{hr}$ ) is an important characteristic for comparison. Pristine fibers began to increase in resistivity at about $350{ }^{\circ} \mathrm{C}$, presumably due to oxidation by atmospheric oxygen. For bromine intercalated fibers, that temperature was about $200{ }^{\circ} \mathrm{C}$, for iodine monochloride intercalated fibers it was about $100{ }^{\circ} \mathrm{C}$, and for cupric chloride intercalated fibers it was somewhat below $100{ }^{\circ} \mathrm{C}$. It was difficult to establish this temperature for ferric chloride intercalated fibers, for upon heating, water was lost and the resistivity went down, confusing the interpretation of the data. But, given the standard state of first drying the fibers for $12 \mathrm{hr}$ in a $200^{\circ} \mathrm{C}$ oven, the ferric chloride intercalated fibers appeared to be stable to $250^{\circ} \mathrm{C}$.

To put these data into an applications perspective, figure 12 shows the stability of the four types of intercalated fibers at $200^{\circ} \mathrm{C}$, which is a typical curing temperature for epoxy resins. Only bromine intercalated fibers retain their resistivity, and, hence, their intercalant. Ferric chloride intercalated fibers lose water, and iodine monochloride and cupric chloride intercalated fibers probably lose intercalant.

At the highest temperatures studied for all four types of intercalated fiber, there were large variations in the resistance ratio from fiber to fiber, with no plateau indicated. When the fibers were heated long enough, the fiber resistance actually exceeded the pristine resistance value. This upward trend in resistance ratio may be indicating deintercalation, or it may be implicating oxidation of the lattice itself (perhaps by some combination of intercalant and ambient oxygen). In the extreme, fibers ashed away completely. This high temperature degradation was most noticeable at $500{ }^{\circ} \mathrm{C}$ 
for bromine and iodine monochloride intercalated fibers, at $450{ }^{\circ} \mathrm{C}$ for ferric chloride intercalated fibers, and at about $300{ }^{\circ} \mathrm{C}$ for cupric chloride intercalated fibers.

Studies of potassium-intercalated, highly oriented pyrolytic graphite have suggested that deintercalation reaction rates are diffusion-controlled, that is, the intercalant migrates from the central section to the surface (ref. 11). The trends in the resistivity ratios, obtained from the data presented in this paper, suggest that the deintercalation mechanism for these acceptor compounds in graphite fibers may not be a simple diffusion-controlled type. Rather than degrading exponentially to some particular resistivity value, presumably approaching that of the pristine fiber, each of these intercalated fiber types seems to have a characteristic resistivity plateau for each temperature. This implies either that each temperature has its own equilibrium intercalant concentration or that an irreversible chemical reaction occurs (such as covalent bromination of the carbon lattice) which robs the lattice of charge carriers, and perhaps puckers the lattice.

Further complicating the matter were preliminary energy dispersive spectroscopy (EDS) studies performed on bromine intercalated fibers heated to temperatures in excess of $500^{\circ} \mathrm{C}$, which indicated that the concentration of bromine was depleated much more slowly than the resistance of the bromine intercalated fibers increased. This result implies that only a small percentage of the bromine present in the fiber actually contributes to the charge transfer. Previous work at this laboratory has shown that the total bromine concentration in the fiber is only 2 to 4 percent by composition (ref. 12). What then is the role of the "passive" bromine? If it acted merely as a neutral spacer, one might expect the bromine to be removed at high vacuum, contrary to the results reported herein. If, on the other hand, the "passive" bromine is largely covalentiy bonded, then only a small fraction of the bromine is actually intercalated. Untll the chemical state of the bromine in these fibers can be clarified, it is likely that the deintercalation mechanism of these compounds will remain unclear.

\section{SUMMARY}

The resistances of graphite fibers intercalated with bromine, iodine monochloride, ferrl chloride, and cupric chloride were monitored periodically while the fibers were subjected to four environmental conditions: ambient. high vacuum, high humidity, and high temperature. Under ambient conditions, the resistance of bromine intercalated fibers remained constant, the resistance of lodine monochloride and cupric chloride intercalated fibers increased slightiy, and the resistance of ferric chloride intercalated fibers increased significantly. At a vacuum of at least $\left(10^{-5}\right.$ tor $\left.r\right)$, the resistance of all four types of intercalated fibers was constant over time. Bromine was the only intercalant that was unaffected by humidity. Iodine monochloride, cupric chloride, and ferric chloride intercalated fibers, respectively, showed increasing susceptibility to water vapor. Both ferric chloride and bromine intercalated fibers were thermally stable to at least $200{ }^{\circ} \mathrm{C}$; both lodine monochloride and cupric chloride intercalated fibers showed increasing resistance for temperatures over $100^{\circ} \mathrm{C}$. Of the four intercalants bromine is the intercalant of choice. Oniy bromine intercalated fibers were stable at ambient conditions, high vacuum, high humidity, and temperatures up to $200^{\circ} \mathrm{C}$. 


\section{REFERENCES}

1. Gaier, J.R., "Stability of Bromine Intercalated Graphite Fibers," NASA TM-86859, 1984.

2. Natarajan, V., Woollam, J.A., and Yavrouian, A., Synthetic Metals, Vol. 8, No. 3/4, Dec. 1983, pp. 291-295.

3. Cowley, J.M., and Ibers, J.A., "The Structures of Some Ferric chlorideGraphite Compounds," Acta Crystallographica, Vol. 9, 1956, pp. 421-431.

4. Oshima, H., Woollam, J.A., and Vavroulan, A., "Metallic Conductivity and Air Stability in Copper Chloride Intercalated Graphite Fibers, "Journal of Applied Physics, Vol. 53, No. 12, Dec. 1982, pp. 9220-9223.

5. Dziemianowicz, T.S., and Forsman, W.C., "Acceptor Intercalated Graphite Fibers: Kinetics, Product Stability and Applications to Composites," Synthetic Metals, Vol. 8, No. 3/4, Dec. 1983, pp. 297-304.

6. Jaworske, D.A., "Dynamics of Graphite Fiber Intercalation: In Situ Resistivity Measurements with a Four Point Probe," NASA TM-86858, 1984.

7. J.A. Woollam, private communication.

8. "Thorne1" P-100 Carbon Fiber Grade VS-0054. Union Carbide Corp., Technical Information Bulletin No. 465-246.

9. Lange, N.A., and Dean, J..A., eds., Lange's Handbook of Chemistry, 12th ed. MCGraw Hi11, New York, 1979, pp. 10-12.

10. Hirt, A.M., and Kazimir, C.J., "Research Study of Bromine and Ferric Chloride Intercalated Graphite Fibers by Scanning Auger Microscope," Structure Probe Inc., Technical Report No. 50335, 1985.

11. Akuzawa, N., Fujisawa, T., Amemiya, T., and Takahashi, Y., "Stability of Potassium-Graphite Intercalation Compounds in An Oxygen Atmosphere," Synthetic Metals, Vol. 7, No. 1/2. Dec. 1983, pp. 57-63.

12. Hung, C-C., "A Micrographic and Gravimetric Study of Intercalation and Deintercalation of Graphite Fibers," NASA TM-87026, 1985. 

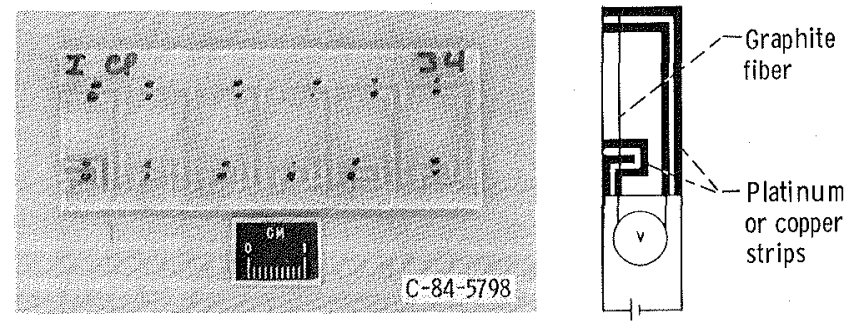

Figure 1. - Four point measurement sample holder of the type used in the stability studies.

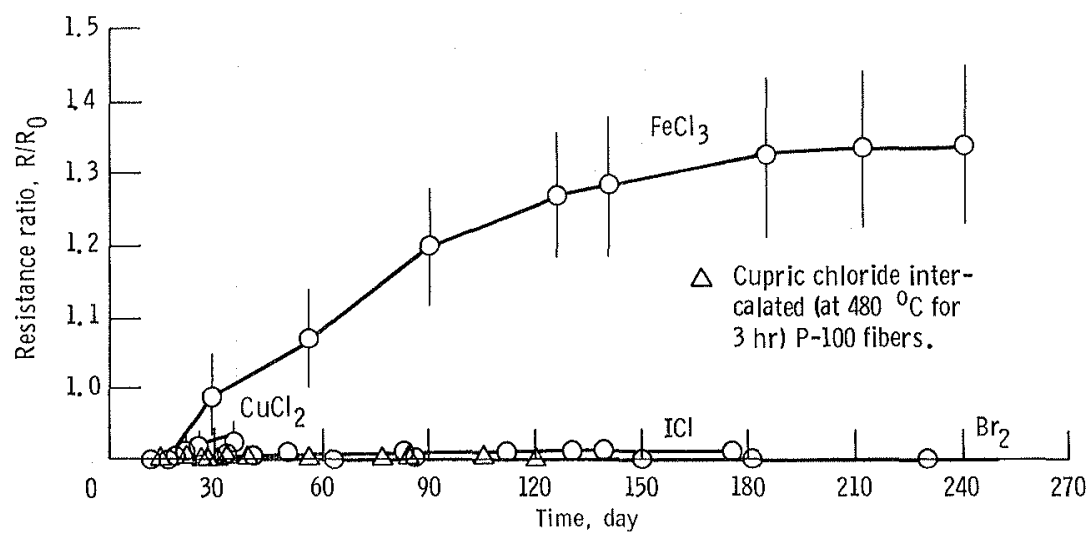

Fig. 2. - Stability of the resistance at ambient laboratory conditions of graphite fibers intercalated with bromine, iodine mionochloride, ferric chloride, and cupric chloride. Vertical lines indicate the observed standard deviation in the resistivity ratio for a group of six fibers. 


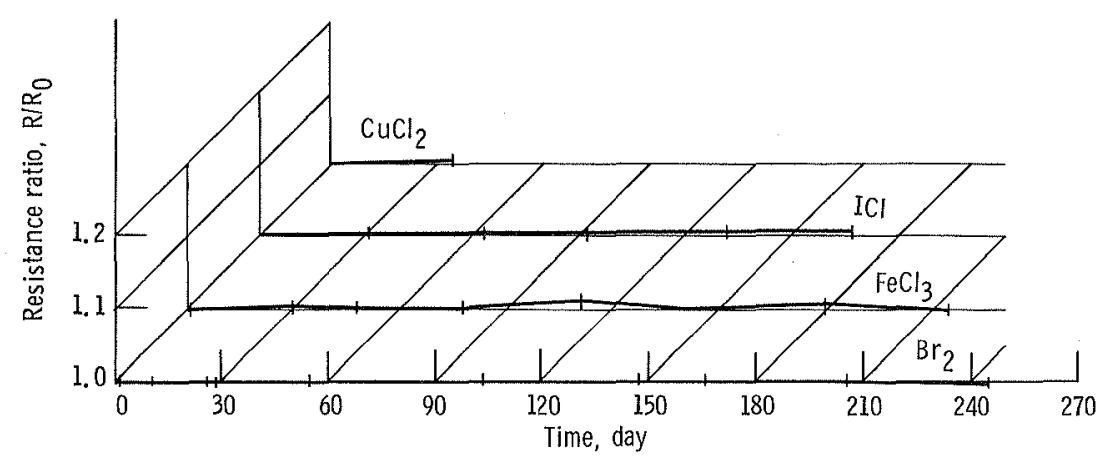

Fig. 3. - Stability of the resistance at vacuum conditions of graphite fibers intercalated with bromine, iodine monochloride, ferric chloride, and cupric chloride.

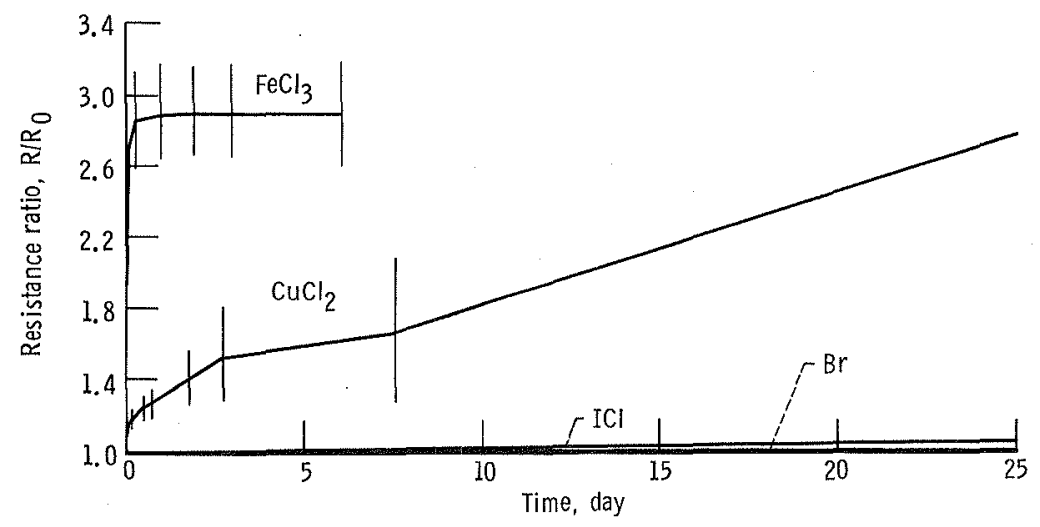

Fig. 4. - Stability of resistance at 100 percent humidlty and $60^{\circ} \mathrm{C}$, conditions of graphite fibers intercalated with bromine, iodine monochloride, ferric chloride, and cupric chloride. 


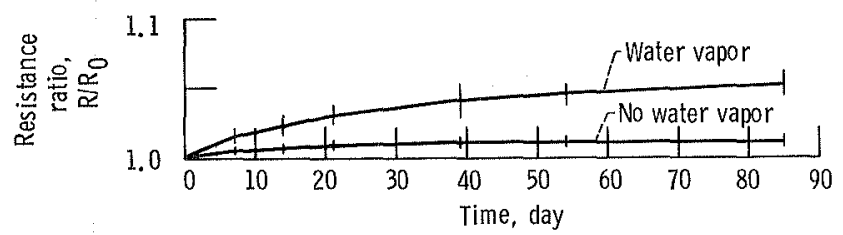

Fig. 5. - Stability of iodine monochloride intercalated graphite fibers at $60^{\circ} \mathrm{C}$ in the absence and presence of water vapor.

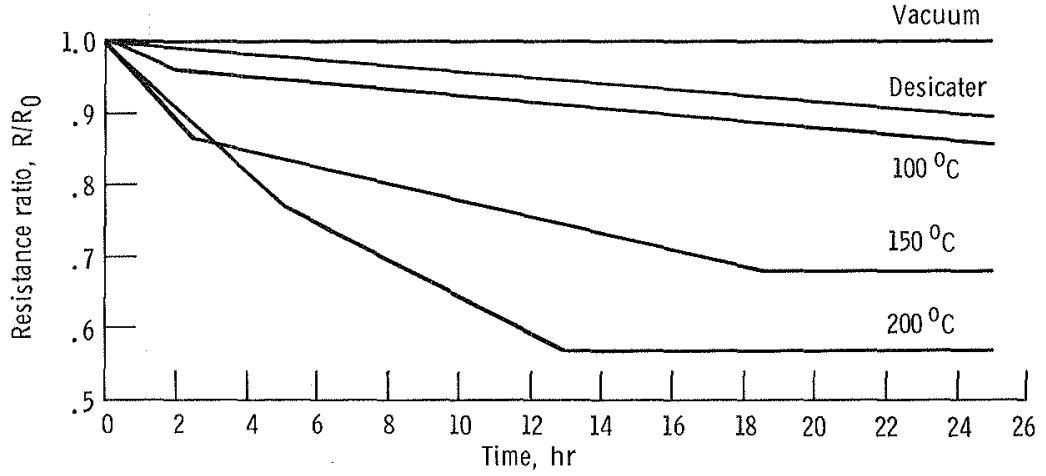

Fig. 6. - Effect of drying techniques on resistance of ferric chloride intercalated graphite fibers. 


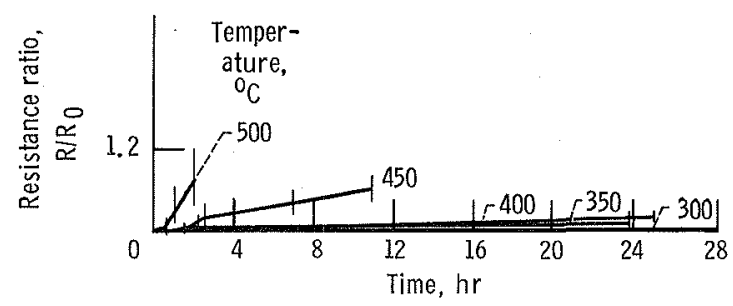

Fig. 7. - Thermal stability of resistance of pristine $\mathrm{P}-100$ fibers in air.

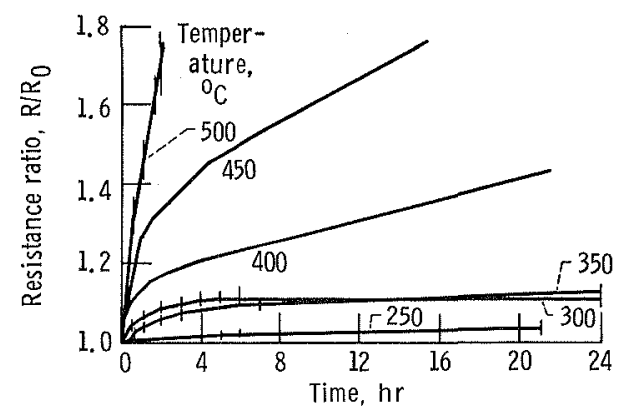

Fig. 8. - Thermal stability of resistance of bromine intercalated graphite fibers in air. 


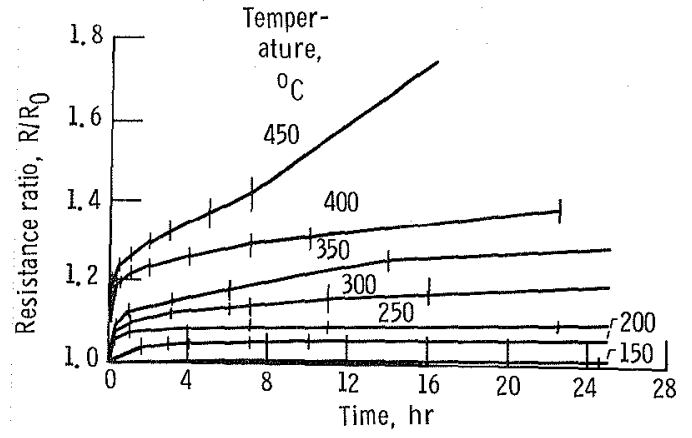

Fig. 9. - Thermal stability of resistance of iodine monochloride intercalated graphite fibers in air.

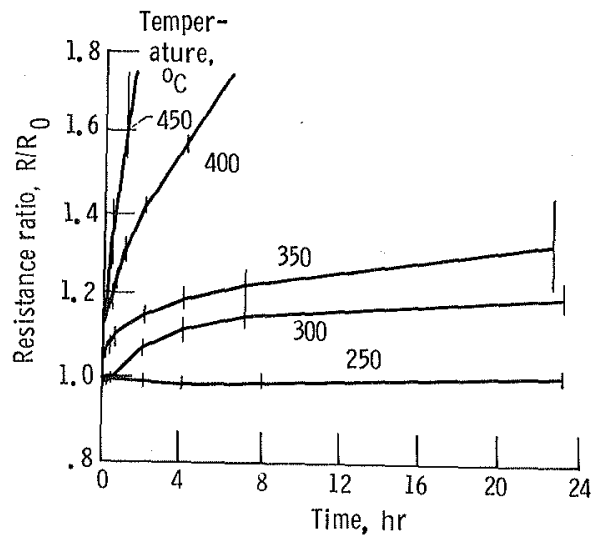

Fig. 10. - Thermal stability of resistance of ferric chioride intercalated graphite fibers in air. 


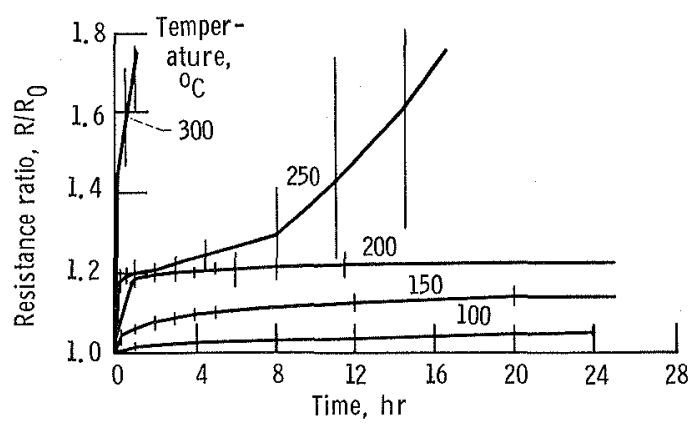

Fig. 11. - Thermal stability of resistance of cupric chloride intercalated graphite fibers in air.

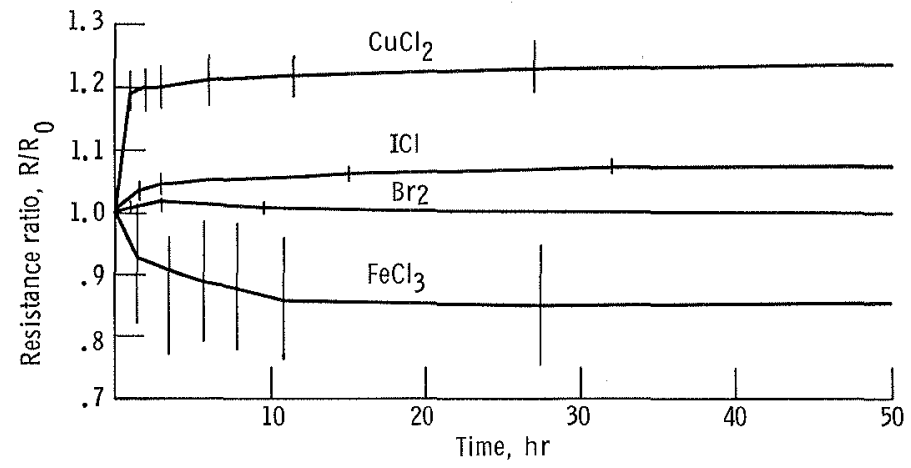

Fig. 12. - Stability of resistance of graphite fibers intercalated with bromine, iodine monochloride, ferric chloride, and cupric chloride at $200^{\circ} \mathrm{C}$. 


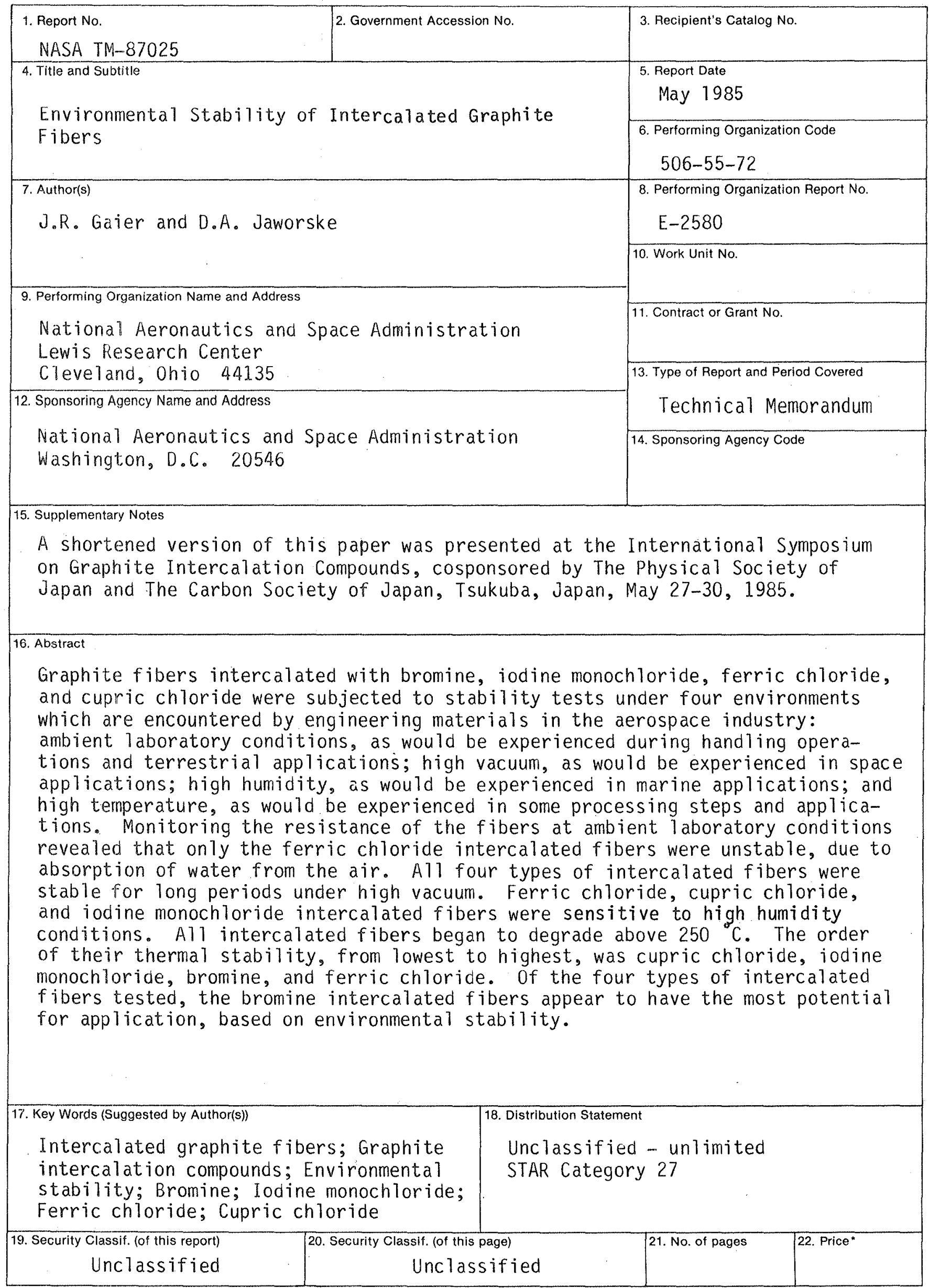

*For sale by the National Technical Information Service, Springfield, Virginia 22161 


\section{End of Document}




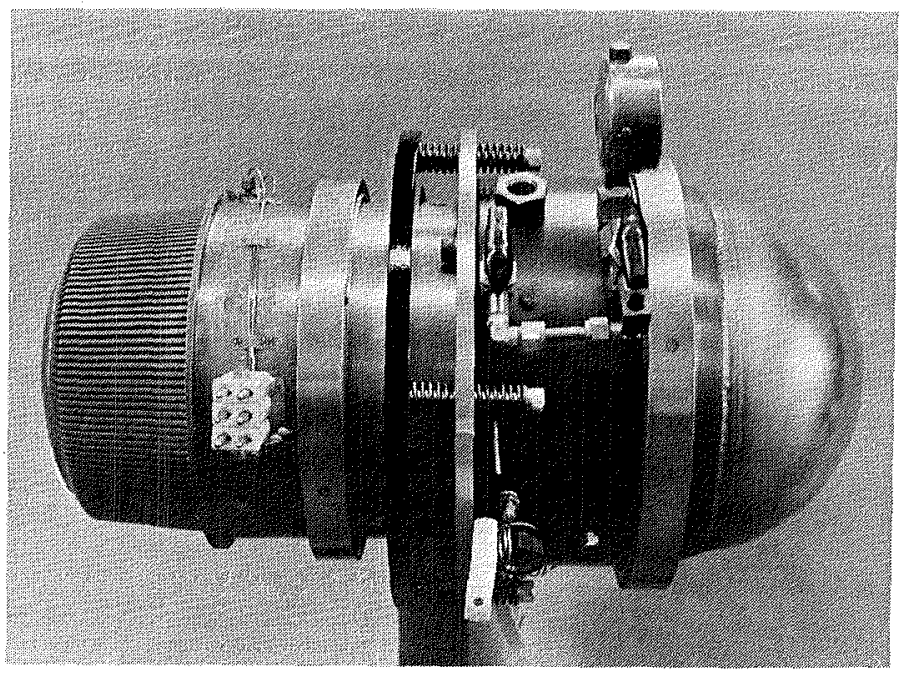

Figure 13. - Sunpower $1 \mathrm{kw}$ free-piston Stirling/linear alternator modified for hydrodynamic gas bearings.

DIMENSIONS

PISTON MASS - KG

BEARING DIAM - D, $M$.

BEARING LENGTH - L, $M$.

BEARING CLEARANCE - $C, M$.

GAS SPRING DIAM - $M$.

GAS SPRING LENGTH - $M$.

GAS SPRING GAP, $M$.

ENGINE FREQUENCY, RAD/SEC

COOLER MASS FLOW RATE, KG/S

PRESSURE, MPa

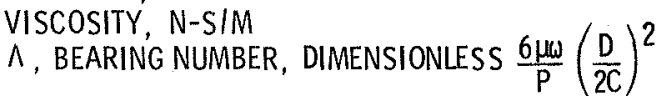

FIPDL, DIMENSIONLESS LOADING

$\varepsilon$, DIMENSIONLESS, ECCENTRICITY RATIO

BEARING $\omega$, RADIS

BEARING POWER LOSS, WATTS

$\mu--$ GAS VISCOSITY

$\omega$ - - ANGULAR VELOCITY

D -- DIAMETER OF BEARING

$P$-- PRESSURE OF BEARING SURROUNDINGS

$C$-- CLEARANCE BETWEEN STATOR AND ROTOR OF BEARING

$F-$ FORCE ON BEARING

$L--$ LENGTH OF BEARING

$\varepsilon-$ ROTOR ECCENTRICITYICLEARANCE

Figure 14. - Proposed scaling of spin bearing test engine.

\section{SPDE TEST ENGINE}

$1.66 \quad 1.45$

$.047 \quad .038$

$.18 \quad .08$

$15 \mathrm{E}-6 \quad 25 \mathrm{E}-6$

$.085 \quad .095$

$.05 \quad .05$

$20 \mathrm{E}-6 \quad 30 \mathrm{E}-6$

$659 \quad 377$

$2.67 \quad .122$

15

$20 \mathrm{E}-6 \quad 20 \mathrm{E}-6$

$.01 \quad .01$

$\begin{array}{cc}.004 & .004 \\ .6 & .6 \\ 500^{6} & 310 \\ 10 & 8\end{array}$




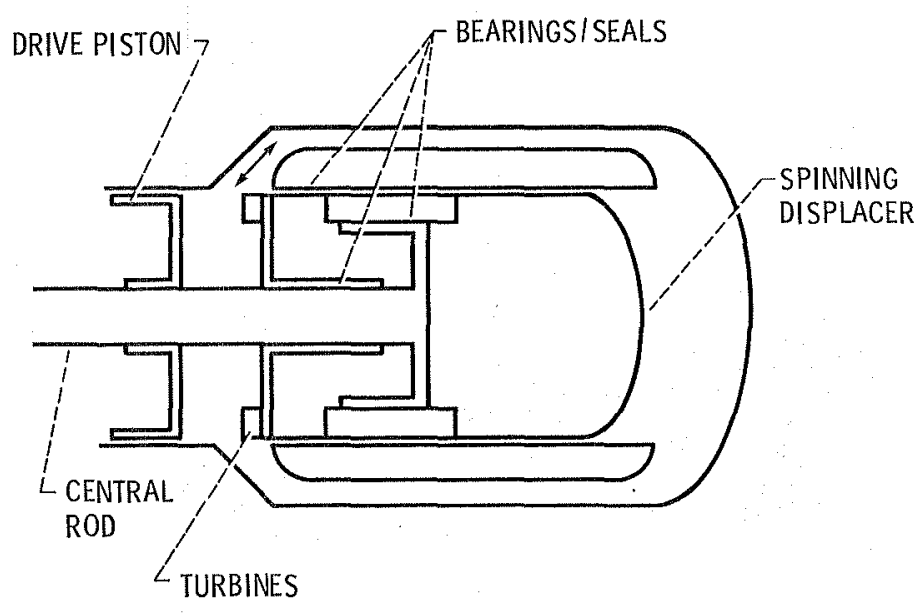

Figure 15. - Spin bearing test rig schematic.

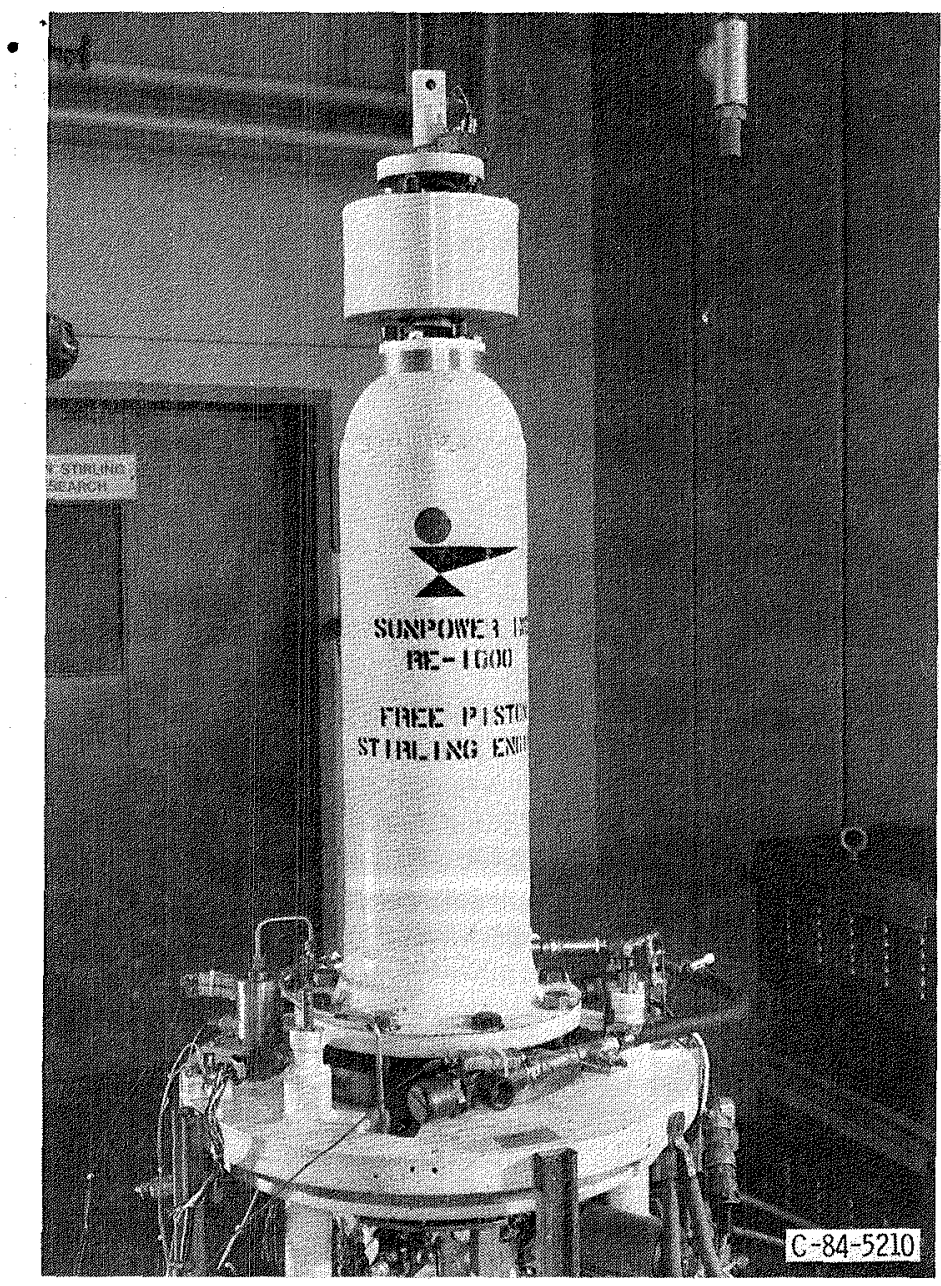

Figure 16. - Balance unit installed on NASAlLewis Re-1000. 


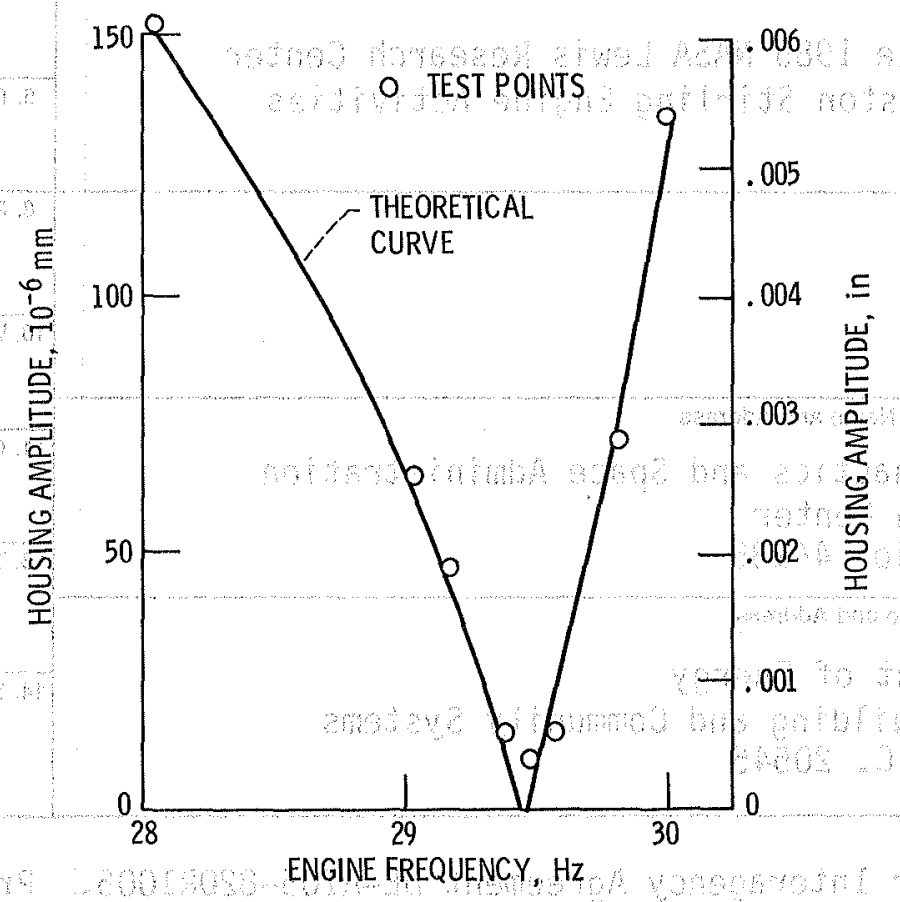

Figure 17. Housing amplitude compared to theoretical value on $\mathrm{RE}-1000$ free-piston Stirling engine.

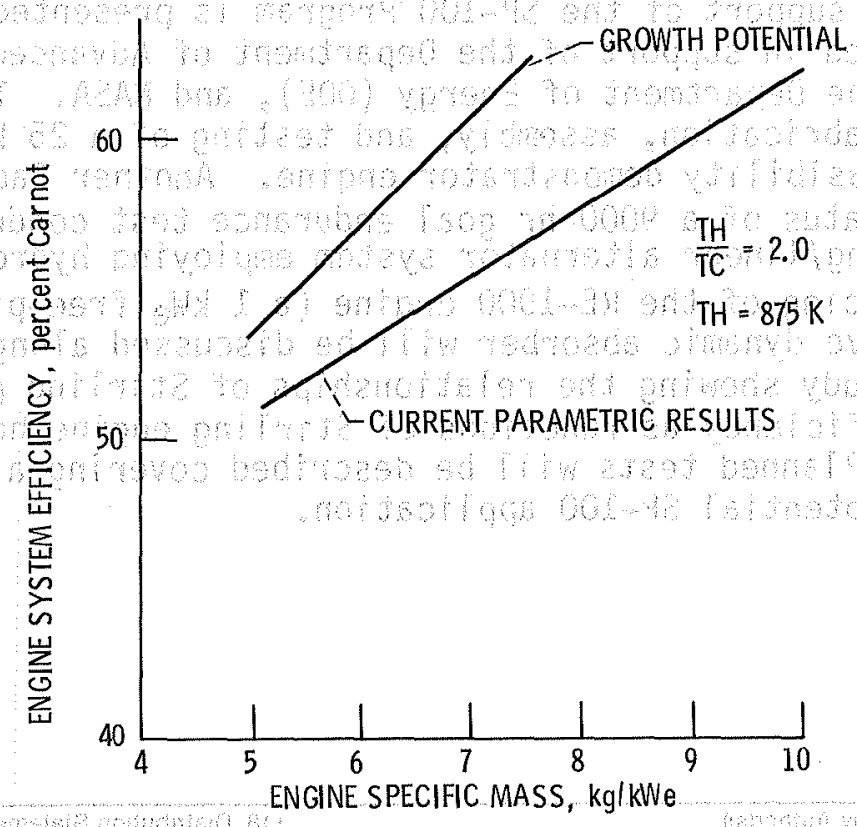

Figure 18. - Potential performance improvements of free-piston Stirling/linear alternator devices. 


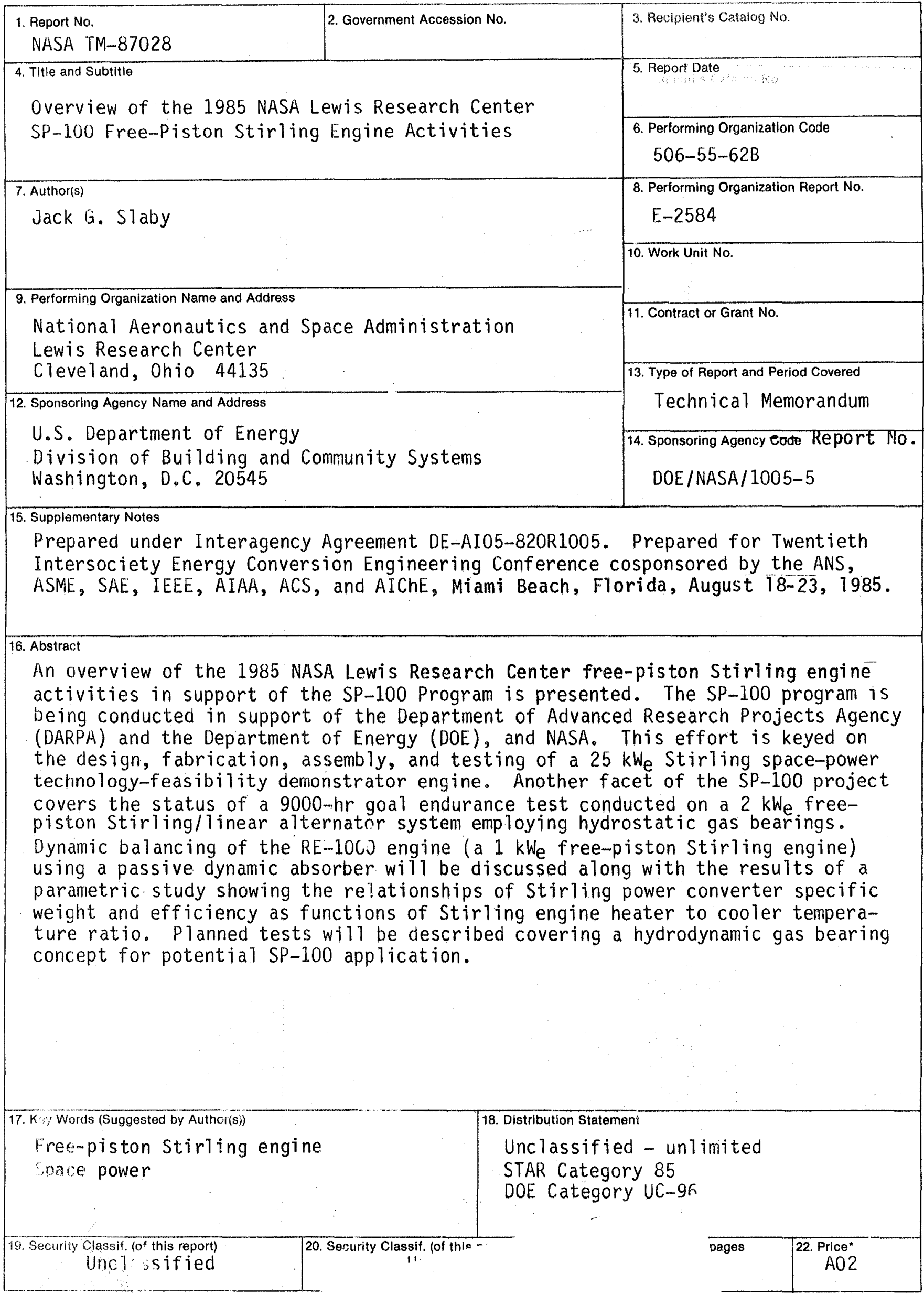

For sicile by the Nationa. 
End of Document 